\title{
A Fault Diagnosis Mechanism for a Proactive Maintenance Scheme for Wireless Systems
}

\author{
Barbara Walsh \\ Centre for Telecommunications Value Chain Research \\ Department of Electronic Engineering \\ National University of Ireland, Maynooth \\ Co. Kildare, Ireland \\ Email: bwalsh@eeng.nuim.ie
}

\author{
Ronan Farrell \\ Institute of Microelectronic and Wireless Systems \\ Department of Electronic Engineering \\ National University of Ireland, Maynooth \\ Co. Kildare, Ireland \\ Email: rfarrell@eeng.nuim.ie
}

\begin{abstract}
This paper presents the fault diagnosis mechanism for a proactive maintenance scheme for wireless systems which is aimed at reducing the high operational costs encountered in the wireless industry by decreasing maintenance costs and system downtime. An on-line monitoring system, based on the aforementioned fault diagnosis mechanism, is used to identify performance degradation, as well as its possible sources, so as to ensure that maintenance occurs only when necessary.
\end{abstract}

\section{INTRODUCTION}

Modern communications systems are becoming increasingly complex so as to meet the burgeoning requirements of consumer applications. The fees at which these services are provided are highly competitive due to the emulous nature of the wireless sector. To maximize profits while meeting requirements such as coverage, capacity and quality of service network operators must take into account financial as well as wireless constraints.

Within these intricate systems there are many possible sources of failure. The RF hardware in particular is quite prone to failure due to the challenging thermal conditions and high power loads to which they are subjected. When failure occurs, the cost for wireless service providers is extensive both in terms of lost revenue and diminishing customer confidence.

In an attempt to reduce the duration of service interruption, considerable measures are taken by the service providers. The principal measure being frequent and often unnecessary preventative maintenance of base stations and antennas. An example of these high network maintenance costs can be seen in Vodafone's published figures [1] for worldwide operational costs. With a total revenue of $£ 14.829$ billion, the total network operating cost is stated as $£ 8.407$ billion, where approximately $50 \%$ of these costs can be attributed to network upkeep.

Elevated maintenance costs are compounded by the lack of standard diagnostics within mobile base stations. Many base station manufacturers make use of third party vendor sub-systems and components when constructing base stations. Often these sub-systems lack self test capabilities or have proprietary diagnostic interfaces. These diagnostic interfaces often provide much information about the status of the system which is neither well understood nor used in a productive manner resulting in generally poor base station diagnostic capabilities.
A considerable burden is placed on the engineering resources of the operator by this multitude of proprietary interfaces which is beyond the capabilities of all but the largest. This inefficiency in terms of diagnostics and hence maintenance, results in unnecessarily high expenditure on the part of the service provider to maintain an level of service which is acceptable to the consumer.

As competition within the wireless communications sector is constantly escalating, both in terms of services offered and fees charged, the reduction of network operation and maintenance costs is extremely desirable. To this end we propose a fault diagnosis mechanism for a proactive maintenance scheme for wireless networks such as those used by wireless service providers which would result in reduced service interruption.

\section{A. Proposed Solution}

An on-line monitoring system with low operational overhead which would monitor the wireless system operation was proposed in [2]. This system monitors any change in performance and any degradation in performance is logged and its cause diagnosed. It was proposed in [2] that performance degeneration diagnosis could be achieved through the analysis of the symbol frequency distribution of the received signal of the communications system. Hence, it would be possible not only to detect and diagnose the cause of failure or degradation before reaching the cell cite, but to minimize the amount of time spent on site carrying out repairs.

This paper provides a more rigorous investigation of the symbol frequency distribution as the diagnosis mechanism of this proactive maintenance system. An analytical method to calculate the symbol frequency distribution is proposed.

As the RF module is often the cause of failure, a failing RF component would be ideal in the initial testing of the proactive maintenance system. Other failure mechanisms will be included at a later date.

The power amplification process in particular is prone to failure. Failure of the PA results in failure of the system. Hence, a PA was selected as the test case for the fault diagnosis mechanism of the proactive maintenance scheme described in this paper. 


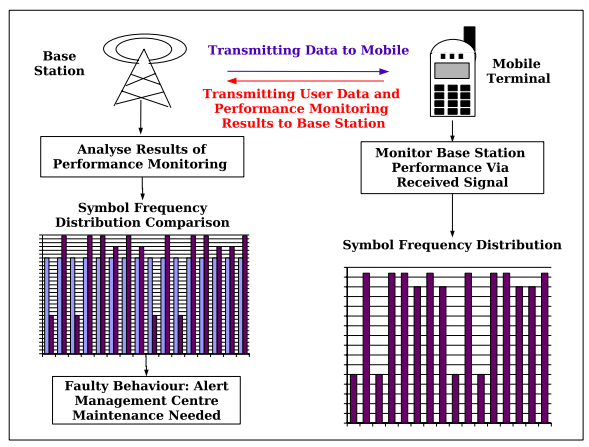

Fig. 1. Proactive Maintenance System Diagram

The paper is organized as follows: Section II gives an overview of the proposed proactive maintenance system. In Section III the theoretical framework for the fault diagnosis mechanism is provided. Results are presented in Section IV while Section V sets forth the conclusions.

\section{System OvervieW}

A block diagram of the proactive maintenance system advocated in this paper can be seen in Fig. 1.

The algorithm used by the proposed proactive maintenance scheme ensures that maintenance occurs only when strictly necessary. It is based on symbol frequency distributions and is as follows:

- Base station transmits data to the mobile terminal.

- Mobile terminal counts the number of times each of the M constellation symbol is received, $S i$, and the total number of symbols received, $S_{R X}$.

- \# $S_{i}$ and $S_{R X}$ are transmitted to the base station by the mobile terminal at regular intervals.

- The base station calculates the symbol frequency of each of the M constellation symbol.

- This symbol frequency distribution, $F_{S}$, is compared to the expected distribution for correct operation, $F_{E X}$.

- If the distributions do not match then the distribution, $F_{S}$, is compared to a set of possible distributions, $F_{K N O W N}$, which represent certain error mechanisms.

- The network management centre is alerted that maintenance is need, with a diagnosis if one is available.

- If the symbol frequency distribution, $F_{S}$, matches the expected distribution, $F_{E X}$, continue to monitor the system.

\section{Fault Diagnosis Mechanism}

The symbol frequency is used as the fault diagnosis mechanism for the proactive maintenance system. The symbol frequency of the received signal is defined as the number of times each of the $\mathrm{M}$ constellation symbols is received, $\# S_{i}$, relative to the total number of symbols received, $S_{R X}$, and is given by Eqn. (1).

$$
F_{S}=\frac{\# S_{i}}{S_{R X}}
$$

It is proposed that the symbol frequency of each individual symbol is effected by nonlinear distortion and that these



Fig. 2. Gray Encoded 16-QAM Signal

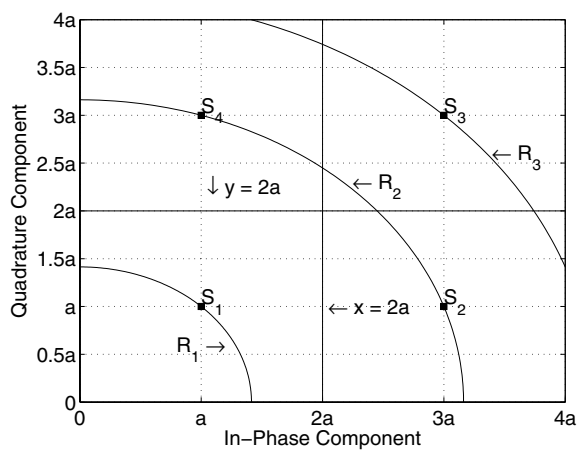

Fig. 3. One Quadrant of a 16-QAM Signal with Decision Boundaries and Symbol Radii

symbol frequencies have a distinctive pattern associated with a particular form of distortion. The theoretical background behind the symbol frequency will be discussed in greater detail in this section for the Gray encoded 16-QAM shown in Fig. 2.

\section{A. Symbol Frequency Distribution for 16-QAM}

The symbols in a 16-QAM signal can be divided into three groups as seen in Fig. 3. Only the first quadrant is shown since the other three quadrants mirror the first one. These groups lie along three radii, $R_{1}=\sqrt{2} a, R_{2}=\sqrt{10} a$ and $R_{3}=3 \sqrt{2} a$. The lines separating the decision regions, $y=2 a$ and $x=2 a$, around these symbols can also be seen. When the signal is subjected to distortion, those symbols which lie on the same radius experience the same level of distortion.

As previously mentioned, the test case for the proactive maintenance system was a PA with degraded linearity performance. To determine the effect of an nonlinear PA on the symbol frequency, the relationship between the decision region boundaries of 16-QAM and the PA model must be considered.

The Rapp Model was used to represent the PA. The AM/AM and AM/PM conversions were applied according to the following functions [3]:

$$
\begin{gathered}
F_{A M / A M}=\frac{u}{\left[1+\left(\frac{u}{V_{S A T}}\right)^{2 p}\right]^{\frac{1}{2 p}}} \\
F_{A M / P M}=0
\end{gathered}
$$




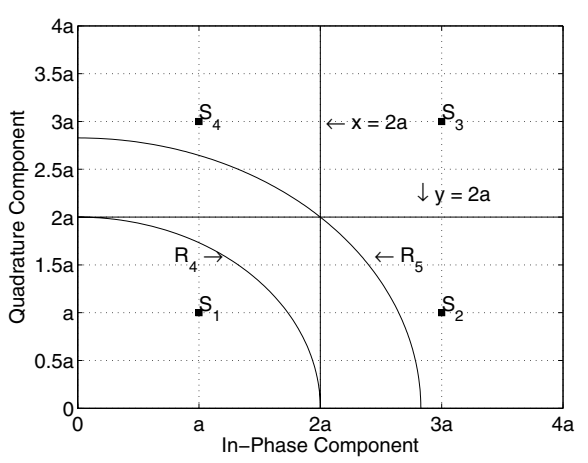

Fig. 4. One Quadrant of a 16-QAM Signal with Decision Boundaries Showing the Effect of Different Levels of $V_{S A T}$

where $u$ is the magnitude of the input signal, $p$ is the smoothness factor and $V_{S A T}$ is the maximum output amplitude.

The value of $V_{S A T}$ effects the level of distortion as is illustrated in Fig. 4. The circles with radii $R_{4}=2 a$ and $R_{5}=2 \sqrt{2} a$ can be seen. The lines separating the decision regions, $y=2 a$ and $x=2 a$, around these symbols can also be seen. When $V_{S A T}<R_{4}$ only $S_{1}$ can be received. When $V_{S A T}<R_{5}$ symbols $S_{1}, S_{2}$, and $S_{4}$ can be received. Finally when $V_{S A T}>R_{5}$ all four symbols can be received.

In the ideal case, where there is no distortion caused by the PA and the effects of the channel are ignored, all symbols would be received with equal probability. Hence, for $V_{S A T}>$ $R_{5}$ :

$$
F_{S_{N}}=\{1 / 16 \quad N=1, \ldots, 16
$$

where $F_{S_{N}}$ is the symbol frequency for a particular symbol and $N=1, \ldots, 16$. For $R_{4}<V_{S A T}<R_{5}$

$$
F_{S_{N}}= \begin{cases}1 / 8 & N=6,8,14,16 \\ 1 / 16 & N=2,4,5,7,10,12,13,15 \\ 0 & N=1,3,9,11\end{cases}
$$

For $V_{S A T}<R_{4}$

$$
F_{S_{N}}= \begin{cases}1 / 4 & N=6,8,14,16 \\ 0 & N=2,4,5,7,10,12,13,15 \\ 0 & N=1,3,9,11\end{cases}
$$

\section{B. Symbol Frequency Distribution for 16-QAM with an AWGN Channel}

In the previous analysis the effects of the channel were ignored. However, the system test case tries to differentiate between a 16-QAM signal with an Additive White Gaussian Noise (AWGN) channel and a 16-QAM signal with an AWGN channel and PA with degraded linearity performance. Hence, the effects of the channel must also be considered when calculating the symbol frequency.

In this case the errors introduced by the channel result in a symbol frequency for each of the $\mathrm{M}$ symbols which is a sum of those symbols which are received correctly as $N$ and those received erroneously as $N, N=1, \ldots, M$. The symbol frequency of a generic M-QAM symbol N, $F_{S_{N}}$, is given by:

$$
F_{S_{N}}=\sum_{i=1}^{M} F_{S_{N, i}}
$$

$F_{S_{N, i}}$ is the frequency with which the symbol $i$ is received as $N$. The component due to those symbols received correctly is calculated as follows:

$$
F_{S_{N_{C O R R E C T}}}=F_{S_{N, N}}=\left(1-P_{E_{N}}\right) \frac{1}{M}
$$

where $P_{E_{N}}$ is the error probability of the symbol $\mathrm{N}$ and is given by [4]:

$$
P_{E_{N}}=\frac{1}{\pi} \sum_{k=1}^{n} \int_{0}^{\theta_{k}} \exp \left[\frac{-X_{k}^{2} \sin ^{2}\left(\Psi_{k}\right)}{2 \sigma^{2} \sin ^{2}\left(\theta+\Psi_{k}\right)}\right] d \theta
$$

where $n$ is the number of sub-regions surrounding the constellation symbol, $\Psi_{k}$ and $\theta_{k}$ are the angles for each of the sub-regions and $X_{k}^{2} / 2 \sigma^{2}$ is a scaled version of the SNR. A more detailed investigation of this error probability analysis and the methodology to calculate all equation parameters can be found in [2].

The component due to those symbols incorrectly received as $N$ may be stated as:

$$
F_{S_{N_{I N C O R R E C T}}}=\sum_{i=1, i \neq N}^{M} F_{S_{N, i}}
$$

where $F_{S_{N, i}}$ is the frequency with which symbols transmitted as $i$ are received as $N . F_{S_{N, i}}$ is calculated as follows:

$$
F_{S_{N, i}}=P_{E_{i, N}} \frac{1}{M}
$$

where $P_{E_{i, N}}$ is the probability that symbol $i$ will be received erroneously as $N$ and is calculated using the relevant element of Eqn. (9).

Hence, the symbol frequency of each of the individual constellation symbols of M-QAM can be generated analytically when the signal is distorted by a nonlinear PA and by an AWGN channel. A particular symbol, $\mathrm{N}$, is a function of both the probability of the sucessful reception of $N$ as well as the erroneous reception of the other sysmbols, $i \neq N$, as the symbol $N$.

\section{RESULTS}

Simulations were carried out carried out in Matlab for a 16QAM signal with an AWGN channel and a 16-QAM signal with a failing PA and an AWGN channel. The failing PA was modeled as an aging amplifier with degraded linearity performance using the Rapp model as previously stated.

Table I gives a sample of how the calculations to find $F_{S_{N}}$ are carried out for symbols 9, 10 and 14 (Fig 3) based on the analysis provided in the previous section. Since the other three quadrants mirror this one the values of symbol frequency also mirror those of this quadrant, for example $F_{S_{9}}=F_{S_{1}}=$ $F_{S_{3}}=F_{S_{11}}$. Furthermore, as symbols 10 and 13 lie on the same radius (Fig. 3), their values are also equal. 
TABLE I

Calculation of Symbol Frequency

$\left(V_{S A T}=3.5 V, p=10, E_{B} / N_{O}=10 d B, V_{S A T}=3 V, p=10\right)$

\begin{tabular}{|c||c||c||c||c|}
\hline$N$ & $P_{E_{N, N}}$ & $F_{S_{N, N}}$ & $\sum F_{S_{N, i}}$ & $F_{S_{N}}$ \\
\hline \hline 9 & 0.1432 & 0.05355 & 0.000125 & 0.053675 \\
\hline 10 & 0.0036 & 0.062275 & 0.00461875 & 0.06689375 \\
\hline 14 & 0.0045 & 0.06221875 & 0.0003 & 0.062518 \\
\hline
\end{tabular}



Fig. 5. Symbol Frequency Comparison Between Calculated and Simulated Results $\left(V_{S A T}=3.5 \mathrm{~V}, p=10, E_{B} / N_{O}=10 \mathrm{~dB}\right)$

Fig. 5 shows both the calculated and simulated symbol frequencies for $V_{S A T}=3.5 \mathrm{~V}, p=10, E_{B} / N_{O}=10 \mathrm{~dB}$. The symbol frequency has 3 distinct levels each of which corresponds to one of the 3 levels of distortion. As can be clearly seen, there is a close match between calculated and simulated result. The calculated and simulated values of the symbol frequency are compared for a range of values of $E_{B} / N_{O}$ with $V_{S A T}=3.5 \mathrm{~V}, 4 \mathrm{~V}, p=10$ as seen in Fig 6 and Fig. 7. Once again there is a good match between calculated and simulated results.

It has been shown that the symbol frequency can be calculated accurately using the methodology provided in this paper for a range of both channel conditions and levels of nonlinear distortion. Hence the use of the symbol frequency as the fault diagnosis mechanism has been validated.

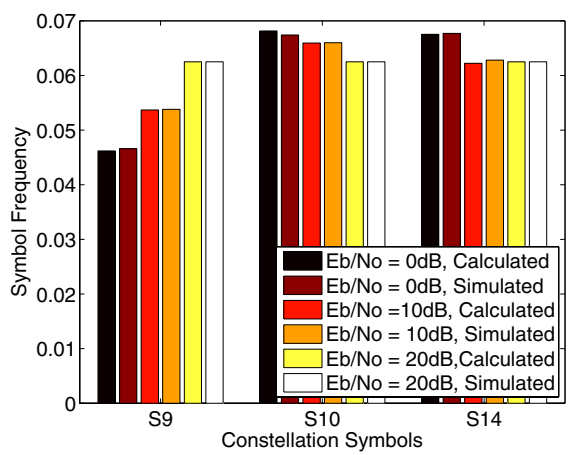

Fig. 6. Symbol Frequency Comparison Between Calculated and Simulated Results $\left(V_{S A T}=3.5 V, p=10, E_{B} / N_{O}=0,10,20 d B\right)$

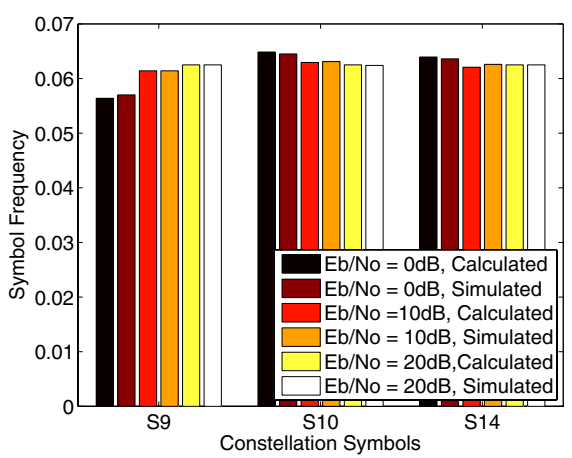

Fig. 7. Symbol Frequency Comparison Between Calculated and Simulated Results $\left(V_{S A T}=4 V, p=10, E_{B} / N_{O}=0,10,20 d B\right)$

\section{CONCLUSion}

Growing user requirements and market competition in the wireless sector intensify the need for reduced operational expenditure by wireless service providers. With this aim in mind the fault diagnosis mechanism for a scheme which would reduce maintenance costs and system downtime has been presented. This system is based on a simple algorithm with low overhead which could be easily implemented. It was shown that this scheme, based on symbol frequency distributions, can be used to identify hardware faults using a PA as the test case. The symbol frequency distribution of the failing PA is easily differentiable from the symbol frequency distribution of an AWGN channel.

An analytical method to calculate the symbol frequency for 16-QAM has been proposed in this paper which is used to verify the simulation results and hence verify the fault diagnosis mechanism.

Generation and verification of symbol frequency distributions for other faults will be carried out in the future. Furthermore a software implementation of the fault diagnosis mechanism will be developed.

\section{ACKNOWLEDGMENT}

The authors would like to thank the Centre for Telecommunications Value Chain Research (CTVR) and the Science Foundation of Ireland for supporting this research.

\section{REFERENCES}

[1] A. Sarin. (2004) Vodafone group plc interim results for the six months to 30 september. financial_results.Par.53582.File.pdf. [Online]. Available: http://www.vodafone.com/start/investor_relations/financial_reports/ previousresults_announcements.html

[2] B. Walsh, G. Baldwin, and R. Farrell, "Physical component performance degradation detection based on error probability analysis," in Proc. IEEE International Symposium on Personal, Indoor and Mobile Radio Communications (PIMRC'07), Athens, Greece, Sept. 2007.

[3] C. Rapp, "Effects of hpa-nonlinearity on a 4-dpsk/ofdm-signal for a digital sound broadcasting system," in Proc. IEEE Second European Conference on Satellite Communications, Liege, Belgium, Oct. 1991, pp. 179-184.

[4] J. W. Craig, "A new, simple and exact result for calculating the probability of error for two-dimensional signal constellations," in Proc. IEEE Military Communications Conference (MILCOM'91), McLean, VA, USA, Nov. 1991, pp. 571-575. 